\title{
Additional Diagnostic Value of Integrated Analysis of Cardiac CTA and SPECT MPI Using the SMARTVis System in Patients with Suspected Coronary Artery Disease
}

\author{
Hortense A. Kirişli*1-3, Vikas Gupta*2,4, Rahil Shahzad*1,3,5, Imad Al Younis ${ }^{6}$, Anoeshka Dharampal ${ }^{3,7}$, \\ Robert-Jan van Geuns ${ }^{7}$, Arthur J. Scholte ${ }^{8}$, Michiel A. de Graaf ${ }^{8,9}$, Raoul M.S. Joemai ${ }^{10}$, Koen Nieman ${ }^{3,7}$, \\ Lucas van Vliet ${ }^{5}$, Theo van Walsum ${ }^{1,3}$, Boudewijn Lelieveldt ${ }^{2,4}$, and Wiro J. Niessen ${ }^{1,3,5}$ \\ ${ }^{1}$ Department of Medical Informatics, Biomedical Imaging Group Rotterdam, Erasmus Medical Center, Rotterdam, The Netherlands; \\ ${ }^{2}$ Division of Image Processing, Department of Radiology, Leiden University Medical Center, Leiden, The Netherlands; ${ }^{3}$ Department \\ of Radiology, Erasmus Medical Center, Rotterdam, The Netherlands; ${ }^{4}$ Department of Intelligent Systems, Faculty of EEMCS, Delft \\ University of Technology, Delft, The Netherlands; ${ }^{5}$ Quantitative Imaging Group, Imaging Science and Technology, Faculty of Applied \\ Sciences, Delft University of Technology, Delft, The Netherlands; ${ }^{6}$ Department of Nuclear Medicine, Leiden University Medical \\ Center, Leiden, The Netherlands; ${ }^{7}$ Department of Cardiology, Erasmus Medical Center, Rotterdam, The Netherlands; ${ }^{8}$ Department of \\ Cardiology, Leiden University Medical Center, Leiden, The Netherlands; ${ }^{9}$ Interuniversity Cardiology Institute of The Netherlands, \\ Utrecht, The Netherlands; and ${ }^{10}$ Department of Radiology, Leiden University Medical Center, Leiden, The Netherlands
}

CT angiography (CTA) and SPECT myocardial perfusion imaging (MPI) are complementary imaging techniques to assess coronary artery disease (CAD). Spatial integration and combined visualization of SPECT MPI and CTA data may facilitate correlation of myocardial perfusion defects and subtending coronary arteries and thus offer additional diagnostic value over either stand-alone or side-byside interpretation of the respective datasets from the 2 modalities. In this study, we investigated the additional diagnostic value of a software-based CTA/SPECT MPI image fusion system over conventional side-by-side analysis in patients with suspected CAD. Methods: Seventeen symptomatic patients who underwent both CTA and SPECT MPI within a 90-d period were included in our study; 7 of them also underwent invasive coronary angiography (ICA). The potential benefits of the synchronized multimodal heart visualization (SMARTVis) system in assessing CAD were investigated through a case study involving 4 experts from 2 medical centers, in which we performed, first, a side-by-side analysis using structured CTA and SPECT reports and, second, an integrated analysis using the SMARTVis system in addition to the reports. Results: The fused interpretation led to a more accurate diagnosis, reflected in an increase in the individual observers' sensitivity and specificity to correctly refer for invasive angiography eventually followed by revascularization. For the first, second, third, and fourth observers, the respective sensitivities improved from $50 \%, 60 \%$, $80 \%$, and $80 \%$ to $70 \%, 80 \%, 100 \%$, and $90 \%$ and the respective specificities from $100 \%, 94 \%, 83 \%$, and $83 \%$ to $100 \%, 100 \%$, $94 \%$, and $83 \%$. Additionally, the interobserver diagnosis agreement increased from $74 \%$ to $84 \%$. The improvement was primarily found in patients presenting with $C A D$ in more vessels than the number of reported perfusion defects. Conclusion: Integrated analysis of cardiac CTA and SPECT MPI using the SMARTVis system results in an improved diagnostic performance.

Received Jan. 23, 2013; revision accepted Jul. 24, 2013.

For correspondence contact: Hortense A. Kirişli, P.O. Box 2040, 3000 CA Rotterdam, The Netherlands.

E-mail: hortense.kirisli@gmail.com

${ }^{*}$ Contributed equally to this work.

Published online Dec. 12, 2013.

COPYRIGHT (C) 2014 by the Society of Nuclear Medicine and Molecular Imaging, Inc.
Key Words: coronary artery disease (CAD); computed tomography angiography (CTA); single-photon emission computed tomography myocardial perfusion imaging (SPECT/MPI); SPECT/CTA fusion; diagnostic and prognostic application

J Nucl Med 2014; 55:50-57

DOI: 10.2967/jnumed.113.119842

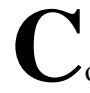
oronary artery disease (CAD) is a major cause of death worldwide (1). Invasive coronary angiography (ICA) is regarded as the reference standard imaging technique for diagnosing CAD (2); it enables determination of the location, extent, and severity of vessel obstructions. CT angiography (CTA) is rapidly gaining clinical acceptance (3); it noninvasively provides high-resolution images of the cardiac and coronary artery anatomy and allows assessment of the presence, extent, and type of coronary stenoses. Still, neither CTA nor ICA provides information on the functional implications of detected stenoses; a functional test may therefore be required to evaluate the presence and extent of myocardial ischemia. SPECT myocardial perfusion imaging (MPI) is widely used to noninvasively assess reversible myocardial ischemia. In conventional side-by-side analysis, integration of CTA and SPECT MPI findings is mentally performed by using a standardized myocardial segmentation model that allocates each myocardial segment to 1 of the 3 main coronary arteries $(4,5)$. However, individual coronary anatomy does not always correspond to the standardized myocardial distribution (6). In a study of 50 patients, Pereztol-Valdes et al. (6) demonstrated that only 9 of the 17 American Heart Association segments are fed by a single coronary artery and that the other 8 segments may be fed by more than one coronary artery. Spatial integration and combined visualization of anatomic and functional data may facilitate correlation of myocardial perfusion defects and subtending coronary arteries and thus offer additional diagnostic value over either stand-alone or side-by-side interpretation of the respective datasets (7-17). The concept of 3-dimensional (3D) fusion imaging to improve 
the assignment of epicardial lesions to stress-induced ischemia originated from the study of Peifer et al. (7) and subsequently of Schindler et al. (8). In the latter, 3D models of the coronary artery tree generated from coronary angiograms were combined with 3D models of the epicardial surface generated from SPECT MPI.

According to more recent studies (9-17), hybrid cardiac imaging systems physically combining the CT and SPECT/PET acquisition, and software allowing fusion of images obtained separately, are promising noninvasive techniques to assess CAD. It is expected that such systems will gain in popularity, to reduce the number of patients unnecessarily referred for ICA examination. Here, we present the software-based synchronized multimodal heart visualization (SMARTVis) fusion system, which allows comprehensive analysis of cardiac multimodal imaging data for assessment of CAD. The aim of the present study was to investigate whether integrated analysis of cardiac CTA and SPECT MPI with the SMARTVis system improves diagnostic performance, compared with side-by-side interpretation.

\section{MATERIALS AND METHODS}

\section{Study Population}

Seventy-one patients who underwent cardiac CTA and SPECT MPI at the Leiden University Medical Center (Leiden, The Netherlands) were randomly selected. After application of exclusion criteria (Fig. 1), 17 patients were included in our study; images from an ICA procedure performed within a 90-d period were available for 7 of them. Patient characteristics are presented in Table 1. The institutional review board approved this retrospective study, and the requirement to obtain informed consent was waived.

\section{SPECT MPI}

SPECT MPI was performed using a 2-d protocol starting with the stress acquisition. The patients underwent bicycle ergometry or, when contraindications were present, adenosine or dobutamine infusion to induce stress. The radioisotope (500 MBq of ${ }^{99} \mathrm{~m}$ Tc-tetrofosmin) was injected at peak exercise, at $3 \mathrm{~min}$ after starting adenosine perfusion, or at peak heart rate during dobutamine infusion. For both stress and rest scans, the images were acquired $1 \mathrm{~h}$ after radioisotope injection.

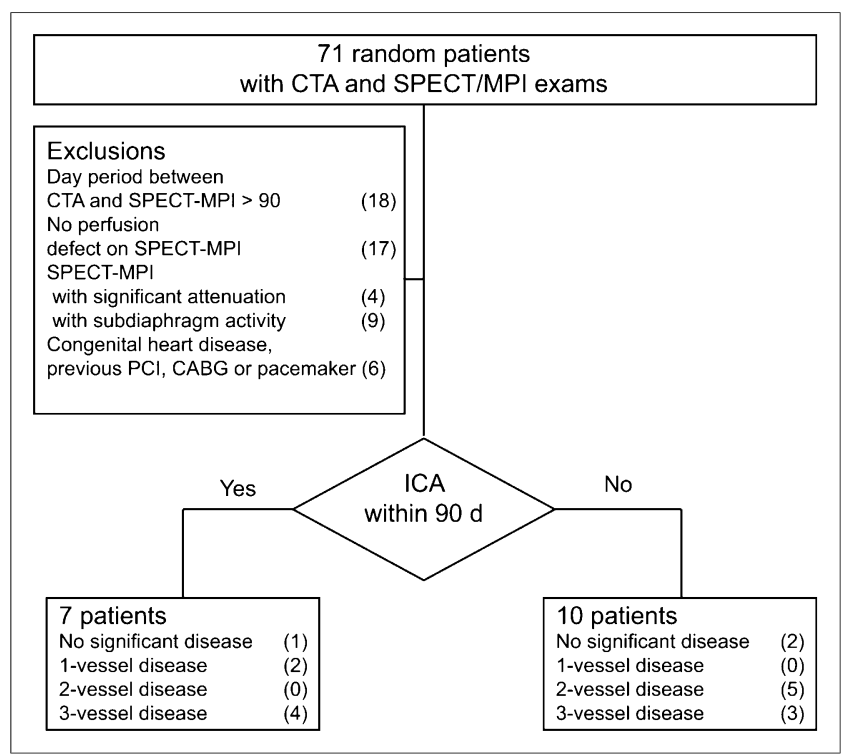

FIGURE 1. Patient inclusion and exclusion criteria. $C A B G=$ coronary artery bypass grafting; $\mathrm{PCl}=$ percutaneous coronary intervention.
A triple-head camera system (CGA 9300; Toshiba) and a low-energy high-resolution collimator were used. Electrocardiographic gating was performed at 16 frames per cardiac cycle, with a tolerance window of $50 \%$. No attenuation or scatter correction was applied.

An experienced nuclear physicist, masked to both CTA and ICA results, analyzed the scans using the Corridor4DM software package (version 6.1; INVIA Solutions) (18). SPECT MPI images were interpreted using oblique slices, polar maps, and quantitative or functional values. The SPECT MPI interpretation was summarized into a report, using the following guidelines (19): the observer graded each of the 19 myocardial segments as being normal (no perfusion defect) or abnormal (reversible or fixed defects) and indicated the extent of myocardial infarction or ischemia.

\section{CTA}

Five patients were scanned using a 64-slice CT scanner (Aquilion 64; Toshiba Medical Systems Corp.), and the remaining 12 patients were scanned using a 320-slice CT scanner (Aquilion ONE; Toshiba Medical Systems Corp.). When the heart rate was higher than 65 beats/min, additional oral $\beta$-blockers (metoprolol, $50 \mathrm{mg}$, single dose, $1 \mathrm{~h}$ before the scan) were provided when tolerated. A prospectively triggered coronary calcium scan (unenhanced CT scan) was performed before the CTA acquisition. CTA images were acquired with a collimation of $64 \times 0.5 \mathrm{~mm}$ for the 64-slice scanner and $320 \times 0.5 \mathrm{~mm}$ for the 320-slice scanner, a tube rotation time of $400 \mathrm{~ms}$, and a tube current of $300 \mathrm{~mA}$ at $120 \mathrm{kV}$ for patients with a body mass index of less than $30 \mathrm{~kg} / \mathrm{m}^{2}$. If a patient had a higher body mass index, tube current was increased to 350 or $400 \mathrm{~mA}$ at $135 \mathrm{kV}$. The acquisition of imaging was prospectively triggered at $75 \%$ of the $\mathrm{R}-\mathrm{R}$ interval. Between 80 and $110 \mathrm{~mL}$ of nonionic contrast material (Iomeron $400 \mathrm{H}$; Bracco Atlanta Pharma) were administered with a flow rate of $5 \mathrm{~mL} / \mathrm{s}$ depending on the total scanning time. The timing of the scan was determined using automated detection of peak enhancement in the aortic root. Th acquisition was conducted during an inspiratory breath-hold of approximately $10 \mathrm{~s}$.

A single experienced reader, masked to both SPECT MPI and ICA results, analyzed the scans using the syngo.via workstation (Siemens Healthcare). CTA images were interpreted using transaxial image stacks and curved multiplanar reformatted images. The CTA interpretation per coronary segment (American Heart Association Model (20)) was summarized into a report, using the following guidelines (21): the observer reported the stenosis location (origin, proximal, mid, distal, or end), the stenosis severity (mild, moderate, severe, or occluded), and the stenosis plaque type (noncalcified, mixed, or calcified).

\section{Quantitative Coronary Angiography (QCA)}

ICA was performed according to standard medical practice. One experienced cardiologist unaware of the CTA and SPECT MPI scoring results performed QCA on the 7 available angiograms. All coronary segments were identified and analyzed using the modified 17-segment American Heart Association classification. Segments were visually classified as normal (smooth parallel or tapering borders, visually $\leq 20 \%$ narrowing) or as having coronary obstruction (visually $\geq 20 \%$ narrowing); the stenoses in these last segments were quantified by a validated QCA algorithm (CAAS; Pie Medical) (22). Stenoses were evaluated in the worst angiographic view and classified as significant if the lumen diameter reduction exceeded $50 \%$.

\section{SMARTVis: A Software-Based CTA/SPECT MPI Fusion System}

In this work, we extended the SMARTVis software-based system that we introduced in 2012 (23) to fuse CTA with SPECT MPI data. An overview of the CTA and SPECT MPI processing and fusion is given in Figure 2. The SMARTVis system provides comprehensive 2-dimensional (2D) and 3D fused visualizations of the anatomic and 
TABLE 1

Patient Characteristics $(n=17)$

\begin{tabular}{lc}
\hline \multicolumn{1}{c}{ Characteristic } & Data \\
\hline Age $(\mathrm{y}$, mean $\pm \mathrm{SD})$ & $61 \pm 9$ \\
Men $(n)$ & $15(90 \%)$ \\
Body mass index $\left(\mathrm{kg} \times \mathrm{m}^{-2}\right.$, & $22.8 \pm 4.4$ \\
$\quad$ mean $\pm \mathrm{SD})$ & \\
Calcium score (Agatston) & 494 \\
Mean & $85-1,319$ \\
Interquartile range & $0-4,797$ \\
Range & $3(17 \%)$ \\
Medical history based on CTA $(n)$ & $2(12 \%)$ \\
No significant disease & $5(29 \%)$ \\
1-vessel disease & $7(42 \%)$ \\
2-vessel disease & \\
3-vessel disease & $7(42 \%)$ \\
Cardiovascular risk factors $(n)$ & $11(65 \%)$ \\
Current smoker & $14(82 \%)$ \\
Hypertension & $12(70 \%)$ \\
Diabetes mellitus & $4(24 \%)$ \\
Hypercholesterolemia & \\
Family history of coronary & \\
vascular disease & \\
Imaging interval (d, mean \pm SD) & \\
Between CTA and SPECT MPI & \\
( $n=17)$ & \\
Between CTA and ICA $(n=7)$ & $45 \pm 31(1,79)^{\star}$ \\
\end{tabular}

*Minimum and maximum intervals are in parentheses.

functional information for relating coronary stenoses and perfusion defect regions. The coronary artery tree extracted from CTA can be projected onto the 2D stress-rest polar map, and similarly, the perfusion information visualized on a 3D stress-rest polar map can be fused with a 3D model of the heart and its coronary artery tree. Furthermore, the SMARTVis system provides a list of automatically detected and quantified coronary artery stenoses (24). To further assist the user in assigning a culprit lesion to a specific perfusion defect, a distance-based estimation of the patient-specific coronary perfusion territories is provided. Last, the $2 \mathrm{D}$ and $3 \mathrm{D}$ polar map views are synchronized with the CTA stenosis findings and images.

\section{Study Design}

The additional diagnostic value of the SMARTVis system to assess CAD was investigated through a case-study evaluation involving 4 experts from 2 medical centers (Erasmus Medical Center, Rotterdam, The Netherlands, and Leiden University Medical Center, Leiden, The Netherlands). An overview of the study design is presented in Figure 3.

During the analyses, for each of the 17 coronary artery segments that had at least one stenosis of at least $20 \%$ on CTA, the observer first had to indicate the correlation between stenoses and perfusion defects. The stenoses in the considered coronary segment could either be related to a myocardial region showing a perfusion defect on SPECT MPI or be considered hemodynamically not significant (i.e., not inducing a perfusion defect). Subsequently, the observer had to indicate the most appropriate therapeutic decision: medical therapy or revascularization of specific coronary segments.

\section{Analysis}

The revascularization decision strategy and target vessel selection of each observer were compared both with the other observers' decisions and with the reference decision derived from interpretation of QCA and SPECT MPI. The diagnostic performance of CTA, SPECT
MPI, and their fusion was compared on a per-vessel basis to determine the therapeutic-decision agreement, as well as the sensitivity and specificity.

For all 17 patients, an interobserver therapeutic-decision agreement percentage was computed per patient $p$ as follows:

$$
\begin{aligned}
\text { Agreement }_{\text {interobs }}^{p} & =\frac{1}{4} \times \sum_{v=1}^{4} \omega_{v} \text { and } \omega_{v} \\
& =\left\{\begin{array}{c}
100 \text { if all observers agree } \\
50 \text { if } 3 \text { observers agree } \\
0 \text { otherwise }
\end{array}\right.
\end{aligned}
$$

with $v$ the main arteries (right coronary artery [RCA], left anterior descending coronary artery [LAD], left circumflex coronary artery [LCX], intermediate branch) and $\omega_{v}$ the observer therapeutic agreement for vessel $v$.

For the subset of 7 patients who underwent ICA, a therapeuticdecision agreement percentage with respect to the QCA/SPECT MPI decision was computed per patient $p$ as follows:

$$
\begin{aligned}
\text { Agreement }_{\mathrm{QCA}}^{p} & =\frac{1}{4} \times \frac{1}{4} \sum_{\mathrm{obs}=1}^{4} \sum_{v=1}^{4} \omega_{\mathrm{obs}, v} \text { and } \omega_{\mathrm{obs}, v} \\
& =\left\{\begin{array}{c}
100 \text { if }, \text { for vessel } v, \text { observers agree } \\
0 \text { otherwise }
\end{array}\right.
\end{aligned}
$$

with the QCA/SPECT MPI decision. Also, the sensitivity and specificity for revascularization of a coronary artery were computed.

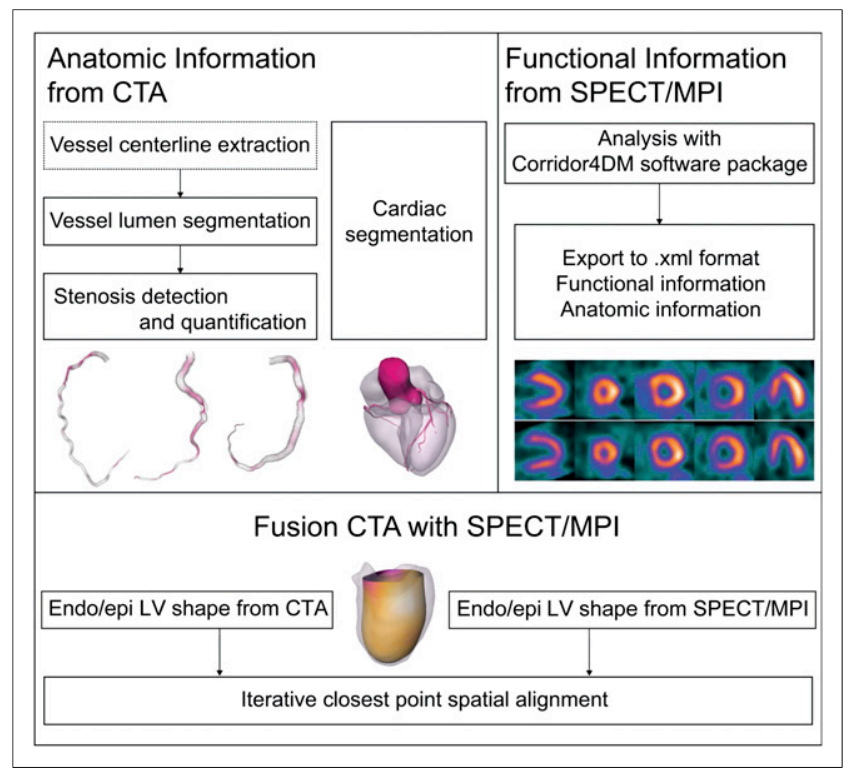

FIGURE 2. Overview of image processing performed on CTA and fusion of CTA/SPECT MPI. Dashed box corresponds to semiautomatic process, whereas solid boxes correspond to fully automatic processes. Previously published methods (24-26) were used to detect and quantify coronary artery stenoses on CTA, quantify coronary calcium, and obtain cardiac chamber shapes from CTA. SPECT MPI left ventricle shape was automatically provided by Corridor4DM software, as were landmark points indicating septal and apical positions. LV shapes and landmark points were subsequently used to align CTA and SPECT MPI data by applying iterative closest-point algorithm. 3D fusion was qualitatively validated by visual inspection of correctness of alignment. Endo/epi = endocardium/epicardium. 


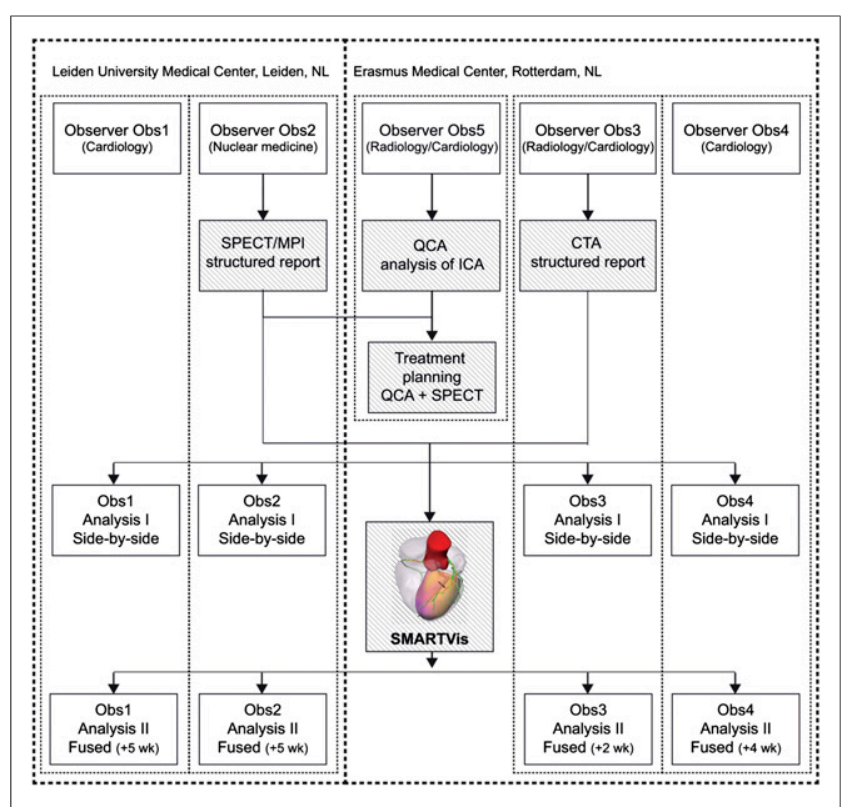

FIGURE 3. Overview of multicenter evaluation study design. First, structured reports were created for CTA and SPECT MPI of 17 patients. QCA analysis was performed for 7 patients. Treatment strategy (i.e., medical treatment or revascularization of specific coronary segments) was further derived from QCA and SPECT MPI findings and served as reference standard. Because guidelines recommend proof of ischemia before revascularization of coronary stenoses (11), expert considered medical therapy indicated for significant lesions detected on QCA that resulted in infarction and no further complaints. During individual sessions, 4 experts examined 17 patients and performed, first, side-by-side analysis using CTA and SPECT MPI reports and, second, integrated analysis using SMARTVis fusion system in addition to reports. Sideby-side analysis was performed $2-5$ wk before integrated analysis to minimize chance of recalling patient cases; patients were analyzed in different order, and expert analyzed several other cases in elapsed time.

\section{RESULTS}

First, the results of the monomodality analyses are reported. Subsequently, we report on interobserver agreement and agreement with the reference standard (combined QCA/SPECT MPI) for the conventional side-by-side analysis and the integrated analysis, respectively. Finally, we compare the performance of integrated analysis of fused CTA/SPECT MPI with the side-byside analysis.

\section{SPECT MPI Findings}

Ten patients $(59 \%)$ showed a reversible perfusion defect; 12 (71\%), a fixed perfusion defect; and 5 (29\%), a mixed perfusion defect. Eight patients $(47 \%)$ showed a perfusion defect in a single coronary territory; 7 (41\%), in 2 territories, and 2 (12\%), in all 3 territories. The exact locations of the perfusion defects are listed in Table 2.

\section{CTA Findings}

Image quality was excellent in 12 patients $(71 \%)$ and moderate in 5 patients (29\%). The median Agatston score was 494 (interquartile range, 85-1,319; range, 0-4,797); 4 patients $(24 \%)$ had a calcium score above 1,000. In total, 263 segments were evaluated, and significant stenoses were present in $66(25 \%)$. The remaining 197 segments $(75 \%)$ were normal or contained only nonsignificant stenoses $(<50 \%)$. Among all segments, 18
(7\%) were qualified as blurred and $6(2 \%)$ were severely calcified. Three patients did not show any signs of CAD. In 2 patients, single-vessel disease was suspected; in 5, double-vessel disease; and in 7, triple-vessel disease. The calcium scores and significant stenosis locations are listed in Table 2.

\section{QCA Findings}

In 7 patients (41\%), conventional ICA was performed within $45 \pm 30 \mathrm{~d}$ after the CTA study. In these 7 patients, 15 of the 100 vessel segments had a stenosis of more than $50 \%$ on ICA, and 26 of the 100 vessel segments had a stenosis of more than $20 \%$ on ICA. One patient did not show any CAD, 1 patient had single-vessel disease, 3 had double-vessel disease, and 2 had triple-vessel disease. The artery segments with at least $20 \%$ stenosis and the QCA values are listed in Table 2. On the basis of the QCA and SPECT MPI findings, revascularization was advised in segments of 10 coronary arteries.

\section{Findings of Side-by-Side Analysis}

Detection of Coronary Lesions Requiring Revascularization. Among the 7 patients for whom QCA was available, there was on average $81 \%$ agreement with regard to the therapeutic decision between the observers and the QCA/SPECT MPI reference standard. Over the 4 (vessels) $\times 7$ (patients) $=28$ therapeutic decisions, the 4 observers agreed in 14 cases (50\%) with the QCA/SPECT MPI therapeutic decision and 3 observers agreed in 9 cases $(32 \%)$. In the remaining 5 cases $(18 \%)$, there was no consensus. For observers 1, 2, 3, and 4, the vessel-based sensitivities to correctly refer for revascularization were $50 \%, 60 \%, 80 \%$, and $80 \%$, respectively, and the vessel-based specificities were $100 \%, 94 \%, 83 \%$, and $83 \%$, respectively.

Interobserver Agreement. Over all patients, the averaged interobserver therapeutic-decision agreement was $74 \%$. Over the 4 (vessels) $\times 17$ (patients) $=68$ therapeutic decisions, the 4 observers agreed in 41 cases $(60 \%)$ and 1 observer disagreed in 19 cases (28\%). For the remaining 8 cases (12\%), no consensus was reached.

\section{Findings of Fused Analysis}

Figures 4 and 5 present an example (patient 8) of visualizations provided by the SMARTVis system for integrated analysis of CTA/SPECT MPI. The other 17 patients are presented in the supplemental data at http://jnm.snmjournals.org.

Detection of Coronary Lesions Requiring Revascularization. For the 7 patients in whom QCA was available, there was on average $91 \%$ agreement with regard to the therapeutic decision between the observers and the QCA/SPECT MPI reference standard. Over the 4 (vessels) $\times 7$ (patients) $=28$ therapeutic decisions, the 4 observers agreed in 20 cases $(72 \%)$ with the QCA/SPECT MPI therapeutic decision and 3 observers agreed in 6 cases $(21 \%)$. For the remaining 2 cases $(7 \%)$, there was no consensus. For observers 1, 2, 3, and 4, the vessel-based sensitivities to correctly refer for revascularization were $100 \%, 90 \%$, $70 \%$, and $80 \%$, respectively, and the vessel-based specificities were $94 \%, 83 \%, 100 \%$, and $100 \%$, respectively.

Interobserver Agreement. Over all patients, the averaged interobserver therapeutic-decision agreement was $84 \%$. Over the 4 (vessels) $\times 17$ (patients) $=68$ therapeutic decisions, the 4 observers agreed in 53 cases $(78 \%)$ and 1 of the observers disagreed in 8 cases $(12 \%)$. For the remaining 7 cases $(10 \%)$, no consensus could be reached.

\section{Comparison of Fused and Side-by-Side Analysis}

Table 3 summarizes the diagnostic performance for the side-byside and fused CTA/SPECT MPI analysis. 


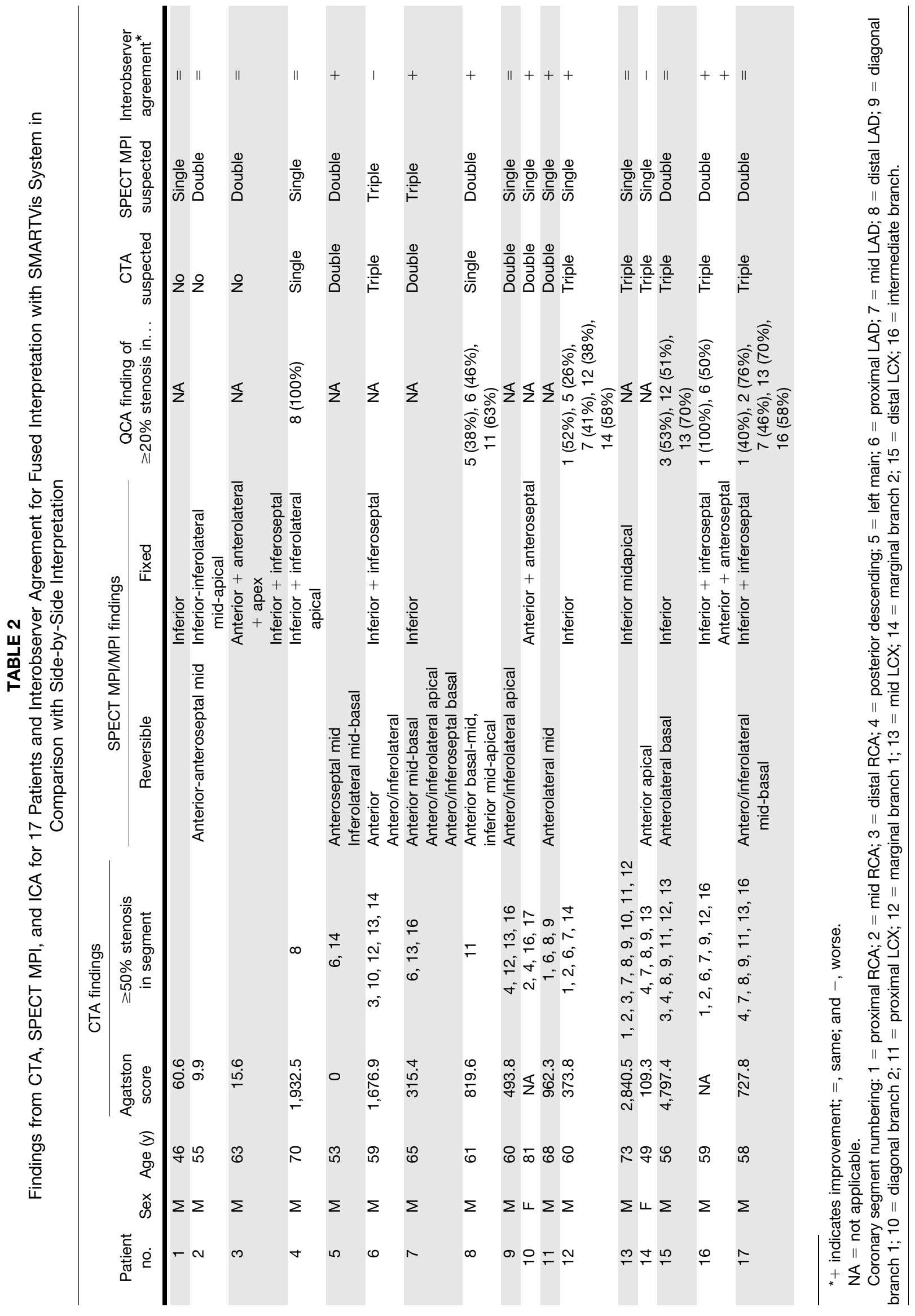

54 The Journal of Nuclear Medicine • Vol. 55 • No. 1 • January 2014 


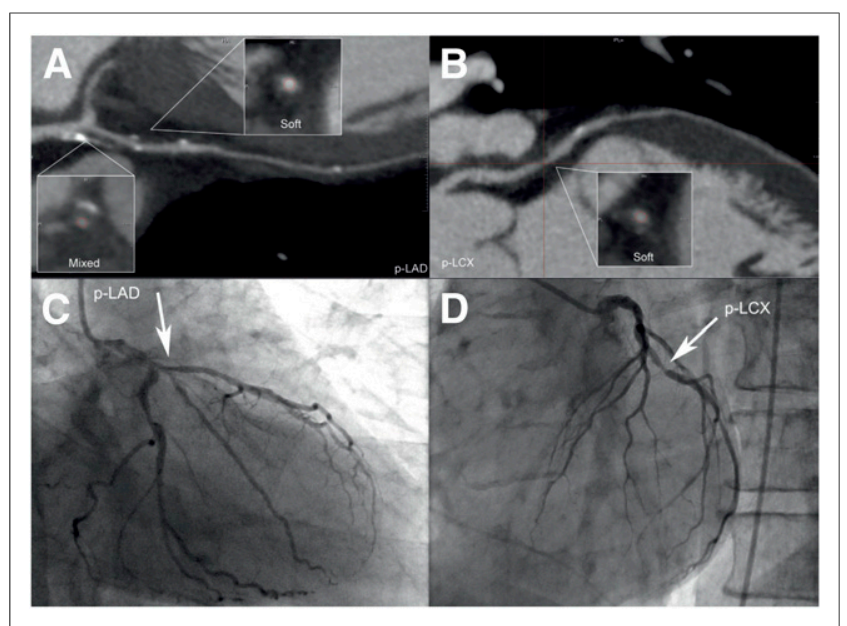

FIGURE 4. CTA and QCA analysis for patient 8, a 61-y-old man. (A and B) CTA report indicates only mild (20\%-50\%) stenoses in LAD (A) and 1 significant stenosis in p-LCX segment (suspected single-vessel disease) (B). (C and D) QCA analysis reveals $46 \%$ stenosis in proximal LAD coronary segment $(C)$ and $63 \%$ stenosis in proximal LCX segment (D). SPECT MPI report indicates 2 reversible perfusion defects in anterior mid-basal and inferior mid-apical walls (suspected double-vessel disease). $\mathrm{p}=$ proximal.

Detection of Coronary Lesions Requiring Revascularization. Through analysis of the integrated SPECT MPI/CTA information using the SMARTVis system, the averaged therapeutic-decision agreement improved in 4 cases (patients 8, 12, 15, and 16) and remained the same in the remaining 3 cases (patients 2, 4, and 17). For all observers, use of the SMARTVis system resulted in an increase of their sensitivity and specificity to correctly refer for revascularization.

Interobserver Agreement. The interobserver therapeutic-decision agreement increased in 8 cases (patients 5, 7, 8, 10, 11, 12, 15, and 16), remained the same in 7 (patients 1, 2, 3, 4, 9, 13, and 17), and decreased in 2 (patients 6 and 14). Over all patients, the interobserver agreement rose from $74 \%$ during the side-by-side analysis to $84 \%$ during the integrated analysis using the SMARTVis system; in the 7 patients who underwent ICA, interobserver agreement increased from $66 \%$ to $82 \%$, suggesting that observer agreement on the more correct therapeutic decisions was also increased using the SMARTVis system.

Territory Disagreement. Disagreement in interpretation between side-by-side and fused analysis was highest for the basal/ mid-inferior/inferolateral myocardial regions (7 patients), which may be supplied by either the RCA or the LCX. Disagreement was also reported for the basal/mid-anterolateral myocardial region (2 patients), to which the $\mathrm{LAD}$, the $\mathrm{LCX}$, or the intermediate branch may supply blood. Last, slight disagreement was noticed in the mid-antero-/inferoseptal myocardial region (1 patient), supplied by either the LAD or the RCA.

\section{DISCUSSION}

Fusion of cardiac CTA anatomic and SPECT MPI functional information for the assessment of CAD was introduced by Nakaura et al. (9). On the basis of 4 cases, the study suggested that fused interpretation improves the relationship between relevant coronary arteries and abnormal perfusion territory. Also, Sato et al. (17) demonstrated that, on the basis of a population of 130 patients, side-by-side combined interpretation of CTA and SPECT MPI provides added diagnostic value, as compared with stand-alone CTA interpretation. Gaemperli et al. (11-13) further investigated the incremental diagnostic value of fused CTA/SPECT MPI interpretation. In one of their studies (11), 38 patients who underwent both CTA and SPECT MPI (25 additionally underwent ICA) and presented with at least 1 perfusion defect on SPECT MPI were included in an evaluation similar to ours (i.e., side-by-side vs. fused). The authors demonstrated that fused analysis provides added diagnostic information on pathophysiologic lesion severity not obtained with sideby-side analysis. The evaluations were performed by a consensus of 2 observers. In our work, 4 independent observers were involved in the evaluation, which allowed us to also investigate the added diagnostic value of fused analysis to reduce interobserver variability in decisions regarding revascularization strategy and target vessel selection. Also, Gaemperli et al. (13) demonstrated that fusion of CTA and SPECT MPI allows accurate detection of flow-limiting coronary stenoses (i.e., significant stenoses inducing ischemia) and that it is thus a potential gatekeeper for ICA and coronary revascularization. In our work, we provide additional insights concerning which patients are more likely to benefit from integrated analysis of fused CTA/ SPECT MPI.

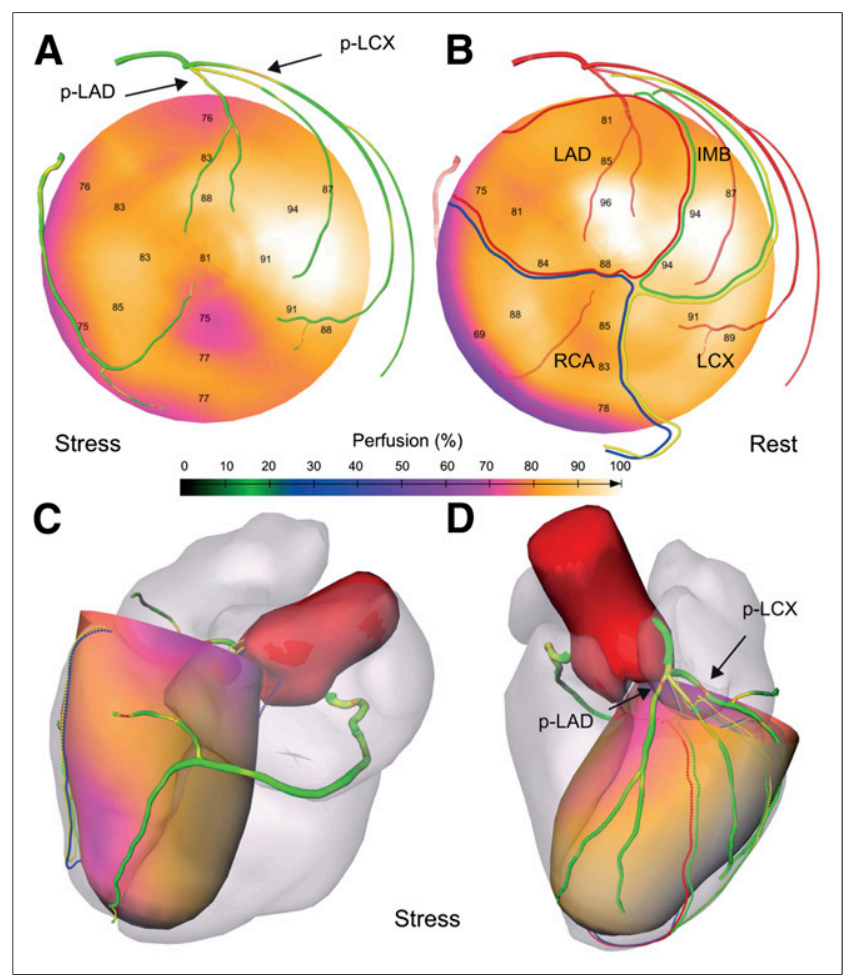

FIGURE 5. Comprehensive visualizations proposed in SMARTVis system for patient 8, a 61-y-old man. (A) 2D stress polar map fused with projection of coronary tree extracted from CTA. Coronary vessel tree is color-coded with automatically estimated degree of stenosis. (B) Rest polar map fused with projection of coronary tree extracted from CTA. Coronary arteries are coded with distance to epicardium: the more transparent the artery, the farther it is from the epicardium. Patient-specific perfusion territories are also projected (LAD in red, LCX in yellow, $\mathrm{MO}$ in green, and RCA in blue). (C and D) 3D model of heart and coronary artery tree extracted from CTA fused with 3D stress polar map. $p=$ proximal. 
TABLE 3

Diagnostic Performance for Side-by-Side and Fused CTAVSPECT MPI Analysis

\begin{tabular}{|c|c|c|c|}
\hline Parameter & No. of patients & Side-by-side & Fused \\
\hline Patients with ICA & 7 & & \\
\hline Agreement with QCA/SPECT MPI & & $81 \%$ & $91 \%$ \\
\hline Interobserver agreement & & $66 \%$ & $82 \%$ \\
\hline Sensitivity (observers 1, 2, 3, and 4) & & $50 \%, 60 \%, 80 \%, 80 \%$ & $70 \%, 80 \%, 100 \%, 90 \%$ \\
\hline Specificity (observers 1, 2, 3, and 4) & & $100 \%, 94 \%, 83 \%, 83 \%$ & $100 \%, 100 \%, 94 \%, 83 \%$ \\
\hline All patients & 17 & & \\
\hline Interobserver agreement & & $74 \%$ & $84 \%$ \\
\hline
\end{tabular}

The results of our case study demonstrated that in several cases the integrated analysis of cardiac CTA and SPECT MPI has a clinical benefit, in the sense that there was both an increase in interobserver agreement and better agreement between therapy planning decisions and the reference standard. Specifically, we found the tool to be of additional value in the diagnosis of patients who have perfusion defects in fewer coronary territories than were suspected to have vessel disease on CTA-that is, who have a perfusion defect in 1 coronary territory and suspected double- or triple-vessel disease on CTA (diagnosis of patients 10, 11, and 12 improved; diagnosis of patients 9 and 13 remained the same) or a perfusion defect in 2 coronary territories and suspected double- or triple-vessel disease on CTA (diagnosis of patients 5, 8, 15, and 16 improved). In such cases, the relation between the coronary territory with a perfusion defect and its supplying coronary arteries is uncertain, thus making the use of the patient-specific SMARTVis system helpful. The case study further revealed that image fusion as implemented in the SMARTVis system has additional diagnostic value neither for patients without coronary stenoses (diagnosis of patients 1,2, and 3 remained the same) nor for patients with suspected single-vessel disease on both CTA and SPECT MPI (diagnosis of patient 4 remained the same) or triple-vessel disease (diagnosis of patient 17 remained the same; diagnosis of patient 6 got worse). In fact, if a patient has no significant stenoses reported but the SPECT MPI study reveals the presence of perfusion defects, the observers consider that the perfusion defects are caused not by obstruction in the epicardial coronary arteries but by microvascular disease or are an artifact. In cases of suspected single-vessel disease, it is clear which coronary artery is causing the perfusion defect, and thus, integrating information in a patient-specific way leads to the same diagnosis as with side-by-side analysis. Also, patients with suspected triplevessel disease do not benefit from such a combined approach. To summarize, integrated analysis of cardiac CTA and SPECT MPI using the SMARTVis system has additional diagnostic value primarily for patients with angiographic CAD that exceeds myocardial hypoperfusion on SPECT MPI.

The results of this work are consistent with the conclusions in previously published work (7-17) on fusion of cardiac CTA and SPECT MPI for assessment of CAD, studies in which fused CTA/ SPECT MPI interpretation appeared to provide added diagnostic information on the hemodynamic relevance of coronary artery lesions. However, our work differs from the previously published work primarily in how the information from CTA and SPECT MPI was fused and how the evaluation was performed. Previously, CTA images were registered (i.e., aligned) with SPECT MPI images to provide fused 3D SPECT/CT images. We introduced a comprehensive visualization system to fuse multimodal imaging data and provide fused representations in both 2 and 3 dimensions for the convenience of the observer. Such an integration of cardiac CTA and SPECT MPI anatomic and functional information into a single coordinated visual analysis tool is novel, maximizing the diagnostic complementarity of CTA and SPECT MPI imaging modalities.

One limitation of our study was the modest population size. However, results were consistent among observers and datasets. A strength was the use of 4 independent observers in the evaluation study. Whether integrated analysis of fused CTA/SPECT MPI using the SMARTVis system is more time-efficient than side-byside interpretation remains to be investigated. The interpretation using the SMARTVis system took from 3 to 15 min, depending on the complexity of the case and on the observer. In the current study, each observer had a training session of only a few minutes using a single excluded patient to become familiar with the SMARTVis system. A reliable investigation of the time efficiency would require a substantially longer use in clinical practice. In general, all observers were enthusiastic about the presented integrated visualization tool and some were eager to use the SMARTVis system in routine clinical practice. Further investigation is needed to determine which patients should undergo such examination (increased imaging costs and radiation dose vs. benefits to the patient). We do not recommend that all patients undergo both CTA and SPECT MPI examination, but we underline that if both tests are performed, integrated analysis is to be preferred.

\section{CONCLUSION}

Integrated analysis of fused cardiac CTA and SPECT MPI using the SMARTVis system results in additional diagnostic value primarily for patients having CAD in more vessels than the number of reported perfusion defects. The SMARTVis comprehensive visualization system can be effectively used to assess disease status in patients with multivessel CAD, offering valuable new options for diagnosis and management.

\section{DISCLOSURE}

The costs of publication of this article were defrayed in part by the payment of page charges. Therefore, and solely to indicate this fact, this article is hereby marked "advertisement" in accordance with 18 USC section 1734. This work was supported in part by a grant from the Dutch Ministry of Economic Affairs (AgentschapNL) under the title "Het Hart in Drie Dimensies" ("Heart in 3D," PID06003). No other potential conflict of interest relevant to this article was reported.

\section{REFERENCES}

1. Roger V, Go A, Lloyd-Jones D, et al. Heart disease and stroke statistics 2012 update: a report from the American Heart Association. Circulation. 2012;125:2-220. 
2. Levine GN, Bates ER, Blankenship JC, et al. 2011 ACCF/AHA/SCAI guideline for percutaneous coronary intervention: a report of the American College of Cardiology Foundation/American Heart Association Task Force on Practice Guidelines and the Society for Cardiovascular Angiography and Interventions. J Am Coll Cardiol. 2011;58:e44-e122.

3. Weustink AC, de Feyter P. The role of multi-slice computed tomography in stable angina management: a current perspective. Neth Heart J. 2011;19: 336-343.

4. Kalbfleisch H, Hort W. Quantitative study on the size of coronary artery supplying areas postmortem. Am Heart J. 1977;94:183-188.

5. Cerqueira MD, Weissman N, Dilsizian V, et al. Standardized myocardial segmentation and nomenclature for tomographic imaging of the heart: a statement for healthcare professionals from the cardiac imaging committee of the Council on Clinical Cardiology of the American Heart Association. Circulation. 2002;105:539-542.

6. Pereztol-Valdés O, Candell-Riera J, Santana-Boado C, et al. Correspondence between left ventricular 17 myocardial segments and coronary arteries. Eur Heart J. 2005;26:2637-2643.

7. Peifer JW, Ezquerra NF, Cooke CD, et al. Visualization of multimodality cardiac imagery. IEEE T Bio-Med Eng. 1990;37:744-756.

8. Schindler TH, Magosaki N, Jeserich M, et al. Fusion imaging: combined visualization of 3D reconstructed coronary artery tree and 3D myocardial scintigraphic image in coronary artery disease. Int J Card Imaging. 1999;15:357-368.

9. Nakaura T, Utsunomiya D, Shiraishi S, et al. Three-dimensional cardiac image fusion using new CT angiography and SPECT methods. AJR. 2005;185:1554-1557.

10. Bax JJ, Beanlands R, Klocke F, et al. Diagnostic and clinical perspectives of fusion imaging in cardiology: is the total greater than the sum of its parts? Heart. 2007;93:16-22.

11. Gaemperli O, Schepis T, Valenta I, et al. Cardiac image fusion from stand-alone SPECT and CT: clinical experience. J Nucl Med. 2007;48:696-703.

12. Gaemperli $\mathrm{O}$, Schepis $\mathrm{T}$, Kalff V, et al. Validation of a new cardiac image fusion software for three-dimensional integration of myocardial perfusion SPECT and stand-alone 64-slice CT angiography. Eur J Nucl Med Mol Imaging. 2007; 34:1097-1106.

13. Gaemperli O, Husmann L, Schepis T, et al. Coronary CT angiography and myocardial perfusion imaging to detect flow-limiting stenoses: a potential gatekeeper for coronary revascularization? Eur Heart J. 2009;30:2921-2929.
14. Santana CA, Garcia E, Faber T, et al. Diagnostic performance of fusion of myocardial perfusion and computed tomography coronary angiography. $\mathrm{J} \mathrm{Nucl}$ Cardiol. 2009;16:201-211.

15. Slomka PJ, Cheng V, Dey D, et al. Quantitative analysis of myocardial perfusion SPECT anatomically guided by coregistered 64-slice coronary CT angiography. J Nucl Med. 2009;50:1621-1630.

16. Kaufmann PA. Cardiac hybrid imaging: state-of-the-art. Ann Nucl Med. 2009;23:325-331.

17. Sato A, Nozato T, Hikita $\mathrm{H}$, et al. Incremental value of combining 64-slice computed tomography angiography with stress nuclear myocardial perfusion imaging to improve noninvasive detection of coronary artery disease. $\mathrm{J} \mathrm{Nucl}$ Cardiol. 2010;17:19-26.

18. Ficaro EP, Lee B, Kritzman J, Corbett J. Corridor4DM: the Michigan method for quantitative nuclear cardiology. J Nucl Cardiol. 2007;14:455-465.

19. Folks RD. Interpretation and reporting of myocardial perfusion SPECT: a summary for technologists. J Nucl Med Technol. 2002;30:153-163.

20. Austen WG, Edwards JE, Frye RL, et al. A reporting system on patients evaluated for coronary artery disease: report of the Ad Hoc Committee for Grading of Coronary Artery Disease, Council on Cardiovascular Surgery, American Heart Association. Circulation. 1975;51:5-40.

21. Raff GL, Abidov A, Achenbach S, et al. SCCT guidelines for the interpretation and reporting of coronary computed tomographic angiography. J Cardiovasc Comput Tomogr. 2009;3:122-136.

22. Reiber JH, Serruys P, Kooijman C, et al. Assessment of short-, medium-, and long-term variations in arterial dimensions from computer-assisted quantitation of coronary cineangiograms. Circulation. 1985;71:280-288.

23. Kirişli HA, Gupta V, Kirschbaum S, et al. Comprehensive visualization of multimodal cardiac imaging data for assessment of coronary artery disease: first clinical results of the SMARTVis tool. Int J Comput Ass Rad. 2012;7:557-571.

24. Shahzad R, Kirişli H, Metz C, et al. Automatic segmentation, detection and quantification of coronary artery stenoses on CTA. Int $J$ Cardiovasc Imaging. 2013;29:1847-1859.

25. Shahzad R, van Walsum T, Schaap M, et al. Vessel specific coronary artery calcium scoring: an automatic system. Acad Radiol. 2013;20:1-9.

26. Kirişli HA, Schaap M, Klein S, et al. Evaluation of a multi-atlas based method for segmentation of cardiac CTA data: a large-scale, multi-center and multivendor study. Med Phys. 2010;37:6279-6291. 